\title{
Evolution of Treatment Strategies for Calciphylaxis
}

\author{
Edward A. Ross
}

Division of Nephrology, Hypertension and Renal Transplantation, University of Florida, Gainesville, Fla., USA

\section{Key Words}

Calciphylaxis · Calcific uremic arteriolopathy · Vascular

calcification

\begin{abstract}
Treatment strategies for calciphylaxis are limited by inadequate understanding of its pathophysiology. Mortality reaches $80 \%$, due to progressive skin ischemia, necrosis and infections. In addition to calcium and parathyroid disorders, hypercoagulability can have a role: primary thrombotic disorders as well as secondary, such as proposed warfarin procoagulant effects. Traditional care addresses the calciumphosphate-PTH axis: minimizing calcium intake, calcimimetics, cautious vitamin D analogs, strict phosphate control, and surgical parathyroidectomy if necessary. Newer approaches focus on extraosseous mineralization: dissolution of calcium deposits, altering osteoprotegerin and NF-кB pathways, and treating macrophage or cytokine-mediated inflammation. Sodium thiosulfate has reported success, and is thought to be due to enhanced calcium solubility and dialysis clearance. Bisphosphonates may have efficacy by lowering bone turnover or a variety of vascular tissue mechanisms. The literature for both agents is very limited, and optimal dosing regimens remain unclear. Patients responsive to a medication will have decreasing pain in days and lesions beginning to heal within approximately 2 weeks. Due to high mortality, early use of combination therapy is advocated, although
\end{abstract}

specific protocols have not been well established. The often dramatic improvements in case-based literature are very encouraging and will hopefully lead to more rigorous studies.

Copyright $\odot 2011$ S. Karger AG, Basel

Calciphylaxis [calcific uremic arteriolopathy (CUA)] is a well-described but poorly understood disorder of high morbidity and mortality (60-80\%) that has limited therapeutic options. As it lacks a clear pathophysiology, the purpose of this review is to explain the proposed mechanisms underlying the established and recently suggested treatments and place them in a framework of a pragmatic approach to the care of these patients (table 1).

The treatment of CUA patients has traditionally revolved around the hypothesis that there is an underlying problem with calcium homeostasis, ultimately resulting in calcification of small vessels, associated tissue ischemia, pain, infarction and ultimately secondary infection with the risk of septic death. It is still not clear, however, whether calcium has a primary etiologic role. As originally described in a 1961 animal model by Selye et al. [1], there is a two-step anaphylaxis-like process wherein there is an initial sensitization [e.g. with excess parathyroid hormone (PTH), calcium, phosphate or vitamin D] and then a systemic (e.g. glucocorticoids) or local (e.g. trauma) challenge that initiates inflammation involving calcification; thus not simply an accelerated dystrophic cal-

\section{KARGER}

Fax +4161306 1234

E-Mail karger@karger.ch

www.karger.com
(ㄷ) 2011 S. Karger AG, Basel

0250-8095/11/0345-0460\$38.00/0

Accessible online at:

www.karger.com/ajn
Edward A. Ross, MD

Division of Nephrology, Hypertension and Renal Transplantation

University of Florida, Box 100224

Gainesville, FL 32610-0224 (USA)

Tel. +1 352273 8822, E-Mail Rossea@medicine.ufl.edu 
Table 1. Physiology-based calciphylaxis treatment strategies

Pathophysiology Therapy

PTH/calcium-phosphate mechanisms

High bone turnover/high iPTH levels

High calcium, phosphate, and calcium $\times$ phosphate levels

Extracellular matrix-associated soft tissue or vascular calcium deposition effects

Other extracellular matrix-associated growth and adhesion factor effects

Hypercoagulability mechanisms

Warfarin-induced reduction in thrombosis inhibitors

(i.e. matrix Gla and Gas-6 proteins)

Medication-induced procoagulant state

Decrease in natural anticoagulant

(i.e. low levels or activity of proteins S or C)

Antiphospholipid syndrome and other hypercoagulability conditions

Endothelial cell dysfunction with prothrombotic or other impaired fibrinolysis/thrombolysis conditions

Imbalanced redox mechanisms

Proinflammation mechanisms

Cytokine release

Macrophage activation

Tissue ischemia mechanisms
Cinacalcet

Vitamin D analogs (i.e. cautious paricalcitol)

Noncalcium-based phosphate binders

Bisphosphonates

Avoid oversuppression of iPTH levels

Surgical parathyroidectomy

Tight control of hyperphosphatemia

Avoid calcium-based phosphate binders

Low calcium dialysate

Pyrophosphate $^{1}$

STS to enhance calcium solubility

Bisphosphonates via multiple intracellular, ligand and crystal pathways Pyrophosphate $^{1}$

Endothelin receptor blockade ${ }^{1}$

Discontinue warfarin

Use alternative anticoagulant

Pyrophosphate ${ }^{1}$

Lower dose or choose alternative agent (i.e. when using calcineurin inhibitors)

Nonwarfarin-based anticoagulation

Consider vitamin $\mathrm{K}$ repletion for deficiency

Treat underlying disorder after complete hematology evaluation

STS via nitric oxide and antithrombotic properties Hyperbaric oxygen (proposed)

STS as antioxidant (i.e. via glutathione)

Bisphosphonates as anti-inflammatory agents

Avoid biopsies unless necessary for diagnosis

STS as vasodilator (i.e. via hydrogen sulfide)

Hyperbaric oxygen

Optimize anemia control

Minimize use of vasopressors because of adverse effects on skin perfusion

Relieve hemodynamically significant arterial occlusive lesions

(i.e. angioplasty, endovascular stents)

Endothelin receptor blockade ${ }^{1}$

Impaired endothelial function and provasoconstriction mechanisms

STS via effects on endothelial and vascular smooth muscle cells

(i.e. NO pathways)

Endothelin receptor blockade ${ }^{1}$

Secondary mechanisms that impair healing

Trauma, infection, malnutrition

Minimize surgical debridement, skin manipulation and trauma

Avoid subcutaneous injections

Meticulous wound care with burn ICU expertise as needed

Skin biopsy only when clinically essential

Appropriate antibiotics

Optimize nutrition, including TPN when indicated

Paraneoplastic mechanisms

Treatment (i.e. of underlying malignancy) not well-described

\footnotetext{
${ }^{1}$ Experimental or proposed therapies, without any human data.
} 
cification. Although adopting the calciphylaxis terminology in their first description of this disorder in man, the 1976 case series by Gipstein et al. [2] did not focus on a sensitization-anaphylaxis sequence of events. That classic manuscript demonstrated remarkable insight into this syndrome and highlighted its occurrence in both dialysis and transplant patients, the distribution of the lesions, wound healing and the possible roles of calcium, phosphate, $\mathrm{PTH}$ and parathyroidectomy.

A common theme of many early reports was spontaneous or easily-induced hypercalcemia (e.g. with the use of calcium-based phosphate binders) in the setting of severe secondary hyperparathyroidism and high bone turnover. Case reports of CUA in nonrenal patients with uncontrolled primary hyperparathyroidism support this mechanism. A calcium-phosphate interaction was also suggested by the syndrome reportedly occurring after severe hyperphosphatemia iatrogenically induced by highdose parenteral nutrition infusions. Many aspects of our current approach to treatment still revolve around the control of those conditions marked by high bone turnover. Inability to handle a calcium load would also occur in the converse situation that has become common in the modern era, in which there is low bone turnover associated with PTH levels that are spontaneously or iatrogenically below the therapeutic window. There have been reports that attempted to quantify the increased risk for CUA based on incremental elevations in serum calcium, alkaline phosphatase or other measures of the PTH axis; however, the series have been so small that they are difficult to extrapolate to general practice.

Inconsistent with a calcium-PTH etiology, however, are other case series in which the serum calcium, phosphate, calcium-phosphate product and PTH levels were unremarkable. Indeed, despite in-hospital meticulous control of their parathyroid-mediated mineral and bone disorder, many patients have unrelenting progression of their necrotic skin lesions. From this viewpoint, the tissue calcium deposits represent an epiphenomenon or secondary process following poorly understood primary or triggering events for the CUA syndrome. Furthermore, there have been no particular patterns of laboratory findings that would explain why some individuals have more of an acral presentation of their lesions, as opposed to those with a more proximal pannicular distribution. The description of intimal hypertrophy in the small (and heavily calcified) vessels from biopsies of affected tissue is an interesting finding supporting a dynamic process in which calcification might not be the inciting event.
Interestingly, among the non-calcium-PTH proposed etiologies are various diseases associated with hypercoagulability. This would explain the well-described association of CUA with warfarin or calcineurin inhibitor therapy. It has been proposed that warfarin (or vitamin $\mathrm{K}$ deficiency) leads to a decrease in the vitamin K-dependent gamma carboxylation of matrix Gla and Gas- 6 proteins, thereby attenuating their normal antithrombotic effects and promoting a procoagulation state [3]. This phenomenon may have confounded the literature in that some reports alleging efficacy of certain pharmaceutical therapies may simply have demonstrated the serendipitous benefits of stopping warfarin. Some authors have suggested CUA patients be evaluated for such primary coagulation disorders as protein $\mathrm{S}$ and $\mathrm{C}$ deficiency, the antiphospholipid syndrome, vitamin $\mathrm{K}$ deficiency, and AT3 deficiency. Other populations at risk for CUA are transplant patients with functioning allografts. Descriptions date back to the earliest reports of calciphylaxis, which were prior to the procoagulation concerns in the calcineurin inhibitor era and without there being a clear association with glucocorticoid use. Although there were early hypotheses that CUA might be associated with aluminum deposition, iron repletion therapy or erythropoietin administration, these have not been confirmed by subsequent analyses.

Study of the entity of nonuremic calciphylaxis has highlighted the associations not just with abnormal coagulation and hyperparathyroidism, but also malignancies, alcoholic liver disease, various connective tissue diseases and corticosteroid use [4]. A potential unifying pathophysiological mechanism revolves around regulation of mineralization pathways involving NF- $\kappa \mathrm{B}$ and osteoprotegerin [5]: calcification being enhanced in disorders that increase receptor activation of the former (i.e. more ligand or fewer inhibitors) or decrease the latter.

These proposed mechanisms highlight the uncertainty as to whether recent advances in experimental models of uremia-associated vascular calcification will ultimately prove to be physiologically appropriate for the study of calciphylaxis. If so, there could potentially be new therapeutic targets directed to vascular smooth muscle cells (i.e. in the dynamic process of their changing to a bone phenotype) or the inhibition of calcium-phosphate interactions. For the former, endothelin has been shown to have many effects on extracellular matrix, growth and adhesions factors beyond its vasoconstrictive properties $[6,7]$. Endothelin-1 gene expression and production is increased in a vitamin D-induced vascular calcification 
model, thus raising the theoretical possibility of pharmaceutical intervention with endothelial receptor blockade [6]; however, there are no data for humans. In terms of the latter, recently there have been elegant studies of using pyrophosphate to inhibit calcitriol-induced vascular extracellular matrix calcification in a uremic rat aorta model $[8,9]$ without adverse effects on bone. This approach is especially appealing in that (unlike the case with bisphosphonates) the skeleton might be spared from decreased mineralization by virtue of bone cells having tissue-nonspecific alkaline phosphatase that would remove the pyrophosphate [9]. Pyrophosphate may also have an important role in warfarin-associated thrombosis. In a rat aorta model, calcification occurring with warfarin-induced low matrix Gla protein levels was exacerbated in the absence of pyrophosphate [10]. There have yet to be therapeutic trials of this agent, which would need to incorporate dosing strategies to address its short half-life (30 min in the rat) [9].

Regardless of whether there is a primary disorder of calcium, PTH or phosphate homeostasis, there has been a consensus that careful control of serum calcium levels is needed since tissue calcification can be a secondary and perhaps disease-perpetuating manifestation. Similarly, regardless of underlying cause, tissue hypoxia (i.e. distal to affected occluded vessels), tissue inflammation and local cytokine release may be potential therapeutic targets. Indeed, without a single etiology having been established over many decades, there is the likelihood that there are multiple disorders that can cause these skin lesions [11] - a frustrating situation for clinicians not knowing which of the limited therapies should be recommended for a particular patient. It also explains the multitude of names for this entity: calciphylaxis, CUA, calcifying panniculitis, metastatic calcinosis cutis and cutaneous gangrene with vascular calcification.

\section{Demographics and Risk Factors}

CUA has been reported more commonly since the 1990s, with some centers describing it occurring in approximately $1 \%$ of dialysis patients regardless of treatment modality, dialysis dose or hemofilter membrane. In the recent decade, the incidence has been reported to be as high as $4 \%$, raising the possibility that it is, for unknown reasons, becoming more common. Risk factors include obesity, diabetes, Caucasian race, female gender and malnutrition (low serum albumin levels). While early descriptions emphasized risk in the elderly, it is now clear that it also affects the young and even pediatric patients. As noted above, populations at risk include those with CKD and kidney transplants.

\section{Presentation and Diagnostic Testing}

The older literature and clinical experience from prior eras of poorly controlled secondary hyperparathyroidism highlighted severe distal ischemia: digit necrosis and infections leading to amputations. Currently, however, the more common presentation appears to be that similar to a proximal symmetrical panniculitis: thighs, buttocks and abdomen, particularly in those with a pannus, and relatively sparing the digits. The proximal presentation has a higher reported mortality $(60-80 \%)$ than the acral pattern (20-30\%). It has been proposed that with an erect posture and the resultant dependent pannus, there would be ischemia-inducing 'stretching' of already compromised small blood vessels in the abdominal wall. Initially, patients typically develop superficial violaceous skin lesions that are painful, hyperesthetic or (initially) pruritic. These can have a tender central erythematous area surrounded by pallor or ecchymoses. Subcutaneous nodules correspond to underlying areas of focal ischemia and can extend beyond the edges of the more obvious skin lesions. More widespread ischemia can produce a mottled or serpiginous skin appearance similar to livedo reticularis. With tissue infarction the lesions become intensely painful, necrotic and hemorrhagic, and spread to contiguous areas. This leads to enlarging areas of skin breakdown and infection. Early on, the pain due to the ischemia can be more prominent, deep (as in abdominal pannus presentation) and intense than anticipated by the appearance of the overlying skin, which may initially be intact. There is a characteristic symmetry, so that the thigh and calf lesions may have a 'kissing' appearance. The subcutaneous induration, even before visible necrosis, can be very prominent and the tissue becomes progressively firm. The latter can be related to involvement of nearby skeletal muscle, with rhabdomyolysis and secondary fibrosis, resulting in a 'woody' consistency. This has been described in the large muscle groups such as buttocks and thighs. Other reported locations of CUA include the heart, lung, pancreas, tongue, eye, and penis. Focal nonulcerated skin plaques have also been described, although one wonders whether these are distinct from the separate entity of dystrophic calcification. Autopsies have demonstrated other tissues with calcification and ischemia, but it is unclear whether these were induced by 
preterminal septic shock: lungs, pancreas, intestine, cardiac valves and peritoneum.

Caregivers are frustrated that efforts to debride the wounds can lead to worsening and further spreading of the lesions. Attempts at skin grafting are futile unless there is an effective therapy for the advancing necrosis. The open wounds are subject to infections by multiple organisms, and not surprisingly patients may suffer from multiorganism bacteremia and septic shock. The use of vasopressors can further compromise skin perfusion. The intensity of the nursing care needed for the extensive skin wounds often prompts transfer of the patient to a burn intensive care unit, which may necessitate sending the patient to a different facility. Controlling the intense pain is often problematic and may prompt high-dose continuous intravenous narcotics. Hemodynamic instability with the sepsis can make intermittent hemodialysis difficult and lead to the use of continuous renal replacement therapy. Withdrawal of medical care has not been uncommon in those cases with severe pain and expanding lesions not responding to treatment.

Because instrumentation of the skin may cause or worsen lesions, there sometimes is a reluctance to even do a biopsy in order to establish a definitive diagnosis. This has been a matter of medical judgment. A compromise that we have pursued is identifying an area of skin bordering an open wound and thereby already at high risk for breakdown. The small- and medium-sized arteries and arterioles of a characteristic size (up to $600 \mu \mathrm{m}$ ) and location (dermis and subcutaneous fat) have calcification of the media, intimal fibrosis and endovascular fibrosis with calcification, intravascular thrombosis and sometimes an associated giant cell reaction. Some authors have commented on arteriolar intimal hyperplasia as being a key finding differentiating these lesions from other biopsies involving calcific atherosclerosis, hypotension and bland ischemia. The tissue associated with these arterial lesions can have thromboses of the capillaries and venules, as well as typical appearances of ischemia, apoptosis and infarction. Some practitioners have chosen to avoid the risk of biopsy in the appropriate clinical setting where the pattern of skin findings appears 'classic'. Nevertheless, we believe there is value in a biopsy in most cases, in that there is a short list of differential diagnoses. Importantly, one needs to also consider biopsies of involved tissues other than skin, such as muscle. Making alternative diagnoses can dramatically alter the therapeutic approach (e.g. plasmapheresis, immunosuppression), and those entities include severe peripheral vascular disease with distal extremity lesions, atheroemboli, autoimmune disease-associated necrotizing vasculitis (e.g. with ANA or ANCA positivity), other necrotizing vasculitides (e.g. hepatitis C-associated), cryoglobulinemia, cryofibrinogenemia and oxalate vasculopathy.

\section{Treatment}

\section{Traditional Therapeutic Approaches}

Below is a description of traditional therapeutic approaches (table 1):

- Optimize control of calcium homeostasis to treat or prevent hypercalcemia and avoid a positive calcium balance: discontinue calcium-based phosphate binders, adjust hemodialysis or peritoneal dialysis dialysate (avoid 'high' and consider 'low' calcium concentrations), discontinue or minimize use of vitamin D analogs (see below). Depending on the serum calcium level and the severity of the CUA, hemodialysate calcium levels as low as 1.5 or $1.0 \mathrm{mEq} / \mathrm{l}$ have been used with appropriate monitoring of serial blood concentrations (e.g. ionized calcium levels) because of possible secondary risks due to hypocalcemia (i.e. arrhythmias). Serum calcium levels can be maintained in the low normal or mildly hypocalcemic range during citrate-based continuous renal replacement therapies by adjusting the calcium repletion rate using infusion protocols based on serial determinations of systemic ionized calcium concentration.

- For hyperparathyroidism, consider emergent parathyroidectomy for severe disease, or aggressive medical control with calcimimetics (e.g. cinacalcet). This would reduce PTH levels per se, as some believe the hormone itself may have a role in CUA, as well as reducing serum calcium and phosphate levels as part of the 'hungry bone syndrome'. Typically, medical PTHcontrol regimens rely on cinacalcet, noncalciumbased phosphate binders and close serial monitoring of hormone levels. Many authors have also included use of active vitamin D analogs (e.g. paricalcitol), but these would need to be administered very cautiously because of the risks of unintentionally inducing hypercalcemia or hyperphosphatemia.

- Adjust medications to resolve unintended pharmacologic overcorrection of hyperparathyroidism, and thereby avoid low bone turnover.

- Aggressive control of hyperphosphatemia with noncalcium-based binders (e.g. sevelamer $\mathrm{HCl}$ or carbonate, lanthanum carbonate) and dietary phosphate restriction. 
Table 2. Simulated daily and weekly dosing regimens for sodium thiosulfate at different dialysis regimens (based on empiric dosing as per simulation 1 for a $70-\mathrm{kg}$ adult)

\begin{tabular}{|c|c|c|c|c|c|c|c|c|}
\hline \multirow[b]{3}{*}{$\mathrm{Q}_{\mathrm{b}}, \mathrm{ml} / \mathrm{min}$} & \multicolumn{8}{|c|}{ Dialyses per week } \\
\hline & \multirow{2}{*}{$\frac{3}{400}$} & \multirow{2}{*}{$\frac{3}{250}$} & \multirow{2}{*}{$\begin{array}{l}4 \\
400\end{array}$} & \multicolumn{2}{|c|}{5} & \multirow{2}{*}{$\frac{6}{400}$} & \multicolumn{2}{|c|}{ CVVHD } \\
\hline & & & & 400 & 400 & & 100 & 100 \\
\hline $\mathrm{Q}_{\mathrm{d}}, \mathrm{ml} / \mathrm{min}$ & 800 & 500 & 800 & 800 & 800 & 800 & 35 & 50 \\
\hline HD time, $h$ & 4 & 4 & 3 & 2.5 & 8 & 2 & continuous & continuous \\
\hline Dose post-HD, g & 25 & 24 & 22 & 18 & 24 & 16 & 24, daily & 35 , daily \\
\hline Weekly dose, g & 75 & 72 & 88 & 90 & 120 & 96 & 168 & 245 \\
\hline
\end{tabular}

CVVHD = Continuous venovenous hemodialysis. With permission from Singh et al. [17].

- Avoid (or relocate) subcutaneous injections, skin trauma and unnecessary skin biopsies or instrumentation.

- Discontinue warfarin and use alternative anticoagulants as needed. Appropriate dosing of the recently developed (i.e. oral) thrombin inhibitors may have a role for patients needing long-term anticoagulation (e.g. those with mechanical heart valves), although these have not been studied in CUA.

- Evaluate for primary hypercoagulability and anticoagulate as indicated, including proteins $\mathrm{C}$ and $\mathrm{S}$ and vitamin $\mathrm{K}$ deficiencies, antiphospholipid syndrome, and AT3 deficiency. Avoid procoagulant medications and supratherapeutic calcineurin inhibitor levels.

- Cautious biopsy to help rule out other differential diagnoses.

- Meticulous wound care, using specialized nursing care (e.g. ICU or burn ICU as appropriate). Surgery clearly has an important role in the treatment of nonviable tissue, but the proviso is that debridement should be kept to a minimum because of poor wound healing. Similar close attention to postamputation wounds is needed.

- Optimize tissue perfusion: avoid hypotension and evaluate for amenable (e.g. by angioplasty or stenting) severe vascular disease, particularly for acral presentations.

- General supportive measures including aggressive pain control, treatment of sepsis, anemia management and optimizing nutrition.

- Consider hyperbaric oxygen therapy, particularly when there is known vascular disease, although evidence of its efficacy is limited to small series. In addition to enhanced oxygen delivery, endothelial cell, fibrinolytic and thrombolytic mechanisms have been hypothesized [5].

- Some authors have considered use of glucocorticoids for early CUA presentations, particularly when the skin is nonulcerated, because of a proposed pathophysiologic role of local tissue inflammation; however, this therapy has in fact been considered a risk factor for developing CUA, and its therapeutic use has not been generally recommended or adopted.

\section{Recent Therapeutic Options}

Intravenous Sodium Thiosulfate Therapy. This approach is based on limited reports of it being used for calcific nephrolithiasis in nonuremic individuals or for tumoral calcinosis in hemodialysis patients [12]. The originally proposed treatment rationale is that this small molecule (normally excreted renally) complexes with calcium and thereby increases the solubility of calcific deposits in CUA, thereby enhancing their hemodialysis clearance. Recently, however, there has been more emphasis on sodium thiosulfate therapy (STS) also having important antioxidant (via enhanced glutathione generation), endothelial cell and nitric oxide mechanisms. The latter could have diverse benefits including decreased vascular smooth muscle cell proliferation, antithrombotic properties and effects on matrix metalloproteinases [7]. The metabolite hydrogen sulfide is also thought to be of benefit due to its vasodilatory properties [13]. STS is administered after a hemodialysis treatment and its efficacy is thought to be related to its presence in the blood (i.e. area under the curve) until its removal by the subsequent dialysis session. Typically, $25 \mathrm{~g}$ is given intravenously over $1 \mathrm{~h}$ after each of the thrice weekly high-flux hemodialysis treatments [14-16]. We feel it is important that the dose be adjusted for dialysis clearance when utilizing more intense or frequent intermittent treatments or for continuous modalities [17] (table 2). Until such time that blood levels are readily available, dosing for various dialysis strategies can be predicted by computerized modeling simulations. Dosing for patients with some preserved re- 
nal function is more problematic and pharmacokinetics have only recently been rigorously described [18]. STS is filtered and rapidly excreted, in that proximal tubular reabsorption is greatly exceeded. Other than suggestions for slow prolonged infusions, there are no specific guidelines for dosing in this subset of CUA patients. Use in peritoneal dialysis patients [16] is another topic with very limited experience. STS therapy is generally considered benign and of relatively low cost. Nausea and vomiting may occur after large-dose infusions. Headache and rhinorrhea have also been described [19]. The anticipated minor worsening of the anion gap metabolic acidosis (due to the small accumulation of thiosulfuric acid produced from sodium thiosulfate) is not considered problematic, especially in that it can be attenuated by appropriate changes in bicarbonate supplementation or dialysate concentration. Combined therapy (e.g. STS with PTH control using cinacalcet and cautious vitamin $\mathrm{D}$ analogs) is considered a reasonable strategy [20]. Clinical improvement in the CUA is anticipated within a 2 -week time frame. In our experience one of the first indicators of response is a decrease in pain in the days following drug initiation, and then wound healing that can ultimately take months (reportedly even greater than a year) of therapy. Cases of CUA in the pediatric or young adult population are rare, but in one report three cases (12 to 21 years of age) responded to STS [21].

Treatment with a Bisphosphonate. There have been a growing number of reports on the efficacy of bisphosphonates [12, 22-24]. Some have proposed its mechanism is calcium-mediated and entirely due to control of high bone absorption, thus possibly being part of a combined cinacalcet, vitamin D analog and PTH-control strategy for controlling high bone turnover. Others, however, have suggested that bisphosphonates have independent therapeutic effects on bone and tissue calcification. The physi-

short-term mortality is unclear. One approach would be to consider these agents in patients with uncontrolled high bone turnover states or in those patients who do not have clinical improvement after approximately 2 weeks of STS therapy. Doses utilized have included etidronate disodium at $200 \mathrm{mg}$ orally each day for 14 days [22, 23], pamidronate at $30 \mathrm{mg}$ intravenously (5 doses over 48 days [24] or 9 doses over approximately 3 months [25]) or alendronate at 35 mg once weekly. Clearance of bisphosphonates by hemodialysis may impact dosing schedules, but this has not been fully investigated. Similar to STS, pain is reportedly improved over days to weeks, followed by wound healing that can take months. A relapse successfully treated with another course of pamidronate has been described [25].

In summary, with CUA having mortality even with comprehensive use of traditional therapies, there has been growing attention to combination regimens utilizing at least one of the more developed medication regimens [26]. It is hoped that a better understanding of the pathophysiology of this disorder will define the subsets (i.e. calcium-PTH vs. hypercoagulability mechanisms) most amenable to treatment with the newer therapies: STS or bisphosphonates. Until such time when there are randomized control trials, it is imperative that clinicians participate in the ongoing calciphylaxis registries (i.e. those in the United Kingdom, Germany and the USA [2729]) so that the science can move beyond the limitations of small series and case reports.

\section{Disclosure Statement}

None. ology of the proposed mechanisms is complex and involves (1) osteoprotegerin and NF- $\mathrm{B}$ pathways [increasing the former and inhibiting expression of the ligand to the latter's activating receptor (RANKL) [5]], (2) PTH-related protein and vascular smooth muscle cell-related effects (decreasing calcification by lowering alkaline phosphatase activity), and (3) inhibition of both phosphate transport and calcium-phosphate crystal formation [11, 23]. There are also possible anti-inflammatory effects involving macrophages, tumor necrosis factor, interleukin and other cytokines. Because these medications can have long-term adverse effects (and are typically contraindicated) on dialysis patients (i.e. already at risk for low bone turnover), their role in treating CUA patients with high

References
1 Selye H, Gentile G, Prioreschi P: Cutaneous molt induced by calciphylaxis in the rat. Science 1961;134:1876-1877.

2 Gipstein RM, Coburn JW, Adams DA, Lee DB, Parsa KP, Sellers A, Suki WN, Massry SG: Calciphylaxis in man. A syndrome of tissue necrosis and vascular calcification in 11 patients with chronic renal failure. Arch Intern Med 1976;136:1273-1280.

3 Danziger J: Vitamin K-dependent proteins, warfarin, and vascular calcification. Clin J Am Soc Nephrol 2008;3:1504-1510.

-4 Nigwekar SU, Wolf M, Sterns RH, Hix JK: Calciphylaxis from nonuremic causes: a systematic review. Clin J Am Soc Nephrol 2008; 3:1139-1143.

5 Weenig RH: Pathogenesis of calciphylaxis: Hans Selye to nuclear factor kappa-B. J Am Acad Dermatol 2008;58:458-471. 
-6 Wu SY, Zhang BH, Pan CS, Jiang HF, Pang YZ, Tang CS, Qi YF: Endothelin-1 is a potent regulator in vivo in vascular calcification and in vitro in calcification of vascular smooth muscle cells. Peptides 2003;24:11491156.

7 Sowers KM, Hayden MR: Calcific uremic arteriolopathy: pathophysiology, reactive oxygen species and therapeutic approaches. Oxid Med Cell Longev 2010;3:109-121.

$\checkmark 8$ O’Neill WC, Lomashvili KA, Malluche HH, Faugere MC, Riser BL: Treatment with pyrophosphate inhibits uremic vascular calcification. Kidney Int 2011;79:512-517.

$\checkmark 9$ Persy VP, McKee MD: Prevention of vascular calcification: is pyrophosphate therapy a solution? Kidney Int 2011;79:490-493.

-10 Lomashvili KA, Wang X, Wallin R, O’Neill WC: Matrix gla protein metabolism in vascular smooth muscle and role in uremic vascular calcification. J Biol Chem 2011;286: 28715-28722.

$\checkmark 11$ Rogers NM, Coates PT: Calcific uraemic arteriolopathy: an update. Curr Opin Nephrol Hypertens 2008;17:629-634.

-12 Phanish MK, Kallarackal G, Ravanan R, Lawson TM, Baboolal K: Tumoral calcinosis associated with pyrexia and systemic inflammatory response in a haemodialysis patient: successful treatment using intravenous pamidronate. Nephrol Dial Transplant 2000; 15:1691-1693.

-13 Sen U, Vacek TP, Hughes WM, Kumar M, Moshal KS, Tyagi N, Metreveli N, Hayden MR, Tyagi SC: Cardioprotective role of sodium thiosulfate on chronic heart failure by modulating endogenous $\mathrm{H} 2 \mathrm{~S}$ generation. Pharmacology 2008;82:201-213.
14 Guerra G, Shah RC, Ross EA: Rapid resolution of calciphylaxis with intravenous sodium thiosulfate and continuous venovenous haemofiltration using low calcium replacement fluid: case report. Nephrol Dial Transplant 2005;20:1260-1262.

15 Brucculeri M, Cheigh J, Bauer G, Serur D: Long-term intravenous sodium thiosulfate in the treatment of a patient with calciphylaxis. Semin Dial 2005; 18:431-434.

16 Cicone JS, Petronis JB, Embert CD, Spector DA: Successful treatment of calciphylaxis with intravenous sodium thiosulfate. Am J Kidney Dis 2004;43:1104-1108.

17 Singh RP, Derendorf H, Ross EA: Simulation-based sodium thiosulfate dosing strategies for the treatment of calciphylaxis. Clin J Am Soc Nephrol 2011;6:1155-1159.

18 Farese S, Stauffer E, Kalicki R, Hildebrand T, Frey BM, Frey FJ, Uehlinger DE, Pasch A Sodium thiosulfate pharmacokinetics in hemodialysis patients and healthy volunteers. Clin J Am Soc Nephrol 2011;6:1447-1455.

19 Raymond CB, Wazny LD: Sodium thiosulfate, bisphosphonates, and cinacalcet for treatment of calciphylaxis. Am J Health Syst Pharm 2008;65:1419-1429.

20 Kyritsis I, Gombou A, Griveas I, Agroyannis I, Retsa K, Agroyannis B: Combination of sodium thiosulphate, cinacalcet, and paricalcitol in the treatment of calciphylaxis with hyperparathyroidism. Int J Artif Organs 2008; 31:742-744.

21 Araya CE, Fennell RS, Neiberger RE, Dharnidharka VR: Sodium thiosulfate treatment for calcific uremic arteriolopathy in children and young adults. Clin J Am Soc Nephrol 2006;1:1161-1166.
22 Hanafusa T, Yamaguchi Y, Tani M, Umegaki $\mathrm{N}$, Nishimura Y, Katayama I: Intractable wounds caused by calcific uremic arteriolopathy treated with bisphosphonates. J Am Acad Dermatol 2007;57:1021-1025.

-23 Shiraishi N, Kitamura K, Miyoshi T, Adachi M, Kohda Y, Nonoguchi H, Misumi S, Maekawa Y, Murayama T, Tomita M, Tomita K: Successful treatment of a patient with severe calcific uremic arteriolopathy (calciphylaxis) by etidronate disodium. Am J Kidney Dis 2006;48:151-154.

24 Monney P, Nguyen QV, Perroud H, Descombes E: Rapid improvement of calciphylaxis after intravenous pamidronate therapy in a patient with chronic renal failure. Nephrol Dial Transplant 2004;19:21302132.

25 da Costa JB, da Costa AG, Gomes MM: Pamidronate as a treatment option in calciphylaxis. J Eur Acad Dermatol Venereol 2008;22:1128-1130.

26 Don BR, Chin AI: A strategy for the treatment of calcific uremic arteriolopathy (calciphylaxis) employing a combination of therapies. Clin Nephrol 2003;59:463-470.

27 The UK calciphylaxis registry. http://www. renal.org/academic/UKCalciphylaxisRegistry.aspx (accessed August 2, 2011).

28 German Calciphylaxis Registry. http://www. klinikumcoburg.de/index.php?id=103\&L=1 (accessed August 2, 2011).

29 Calciphylaxis registry. KU Medical Center, The University of Kansas. http://www2. kumc.edu/calciphylaxisregistry/ (accessed August 2, 2011). 